\title{
Variability of the differential rotation of the photospheric magnetic field through solar cycles
}

\author{
Elena Gavryuseva \\ Istituto Nazionale di Astrofisica - Arcetri Astrophysical Observatory, \\ Largo Enrico Fermi 5, I-50125 Florence, Italy \\ email: elena@arcetri.astro.it
}

\begin{abstract}
Differential rotational rate of the magnetic field measured in the photosphere was calculated and its temporal dependence was studied using two independent methods. It was found that the rotational rate has a character of torsional waves running to the equator with 11 year periodicity. At high latitudes the rotation is getting slower during minimum of activity when the field measured there is stronger.
\end{abstract}

Keywords. Sun: magnetic fields, photosphere, activity

We have used the observations of the WSO from http://wso.stanford.edu/synoptic.html of the photospheric field taken from 1976 to 2005. Two independent methods were applied to evaluate the period of the rotation of the solar magnetic field: spectral analysis (FFT) and autocorrelation. In the upper plot of Fig. 1 is shown the sideral period of the magnetic field rotation revealed by the FFT method for the sub-sets of $40 \mathrm{CR}$ (about 3 years) long as a function of time and latitude. The well known differential rotation appears also for the large scale solar magnetic field. Additionally in the sub-polar zones there is a clearly visible decrease of the rotational rate in 1985 and 1994 during solar activity minima and an increase of the rotation rate approximately in 1990 and in 1991 after the polarity inversion. This happens with the 11-year periodicity. The torsional waves firstly discovered by Howard and LaBonte, 1980 in sunspot rotation are present in the magnetic field rotation rate as well up to high latitudes as it is seen on the bottom plot of Fig. 1. The 11-year variability of the deviations of the period from the mean one in the sub-polar zones correspond to the torsional waves. The rotational rate of the pre-equatorial zones varies in time with a periodicity of $55-60 \mathrm{CR}$ about ( $4-5$ years).

The sideral rotational period of the SMF deduced from the full sets of 29 years long (composed of 27721 points) and as a mean of the sub-sets rotation has a 0.5-0.7-day decrease of the period at latitudes higher than 56-60 degrees in both the hemispheres, corresponding to the 1.7-2.3\% level. The accuracy of the autocorrelation method for the full data sets is limited by the longitudinal resolution of 5 degrees, and its accuracy is equal to $1.3 \%$ at most. This result coincides with the latitudinal dependence of the rotation rate calculated by the FFT method for the full data sets, and with the rotation rate calculated by both methods as a mean of the rotation rates corresponding to the shorter subsets. The accuracy of the mean rotation rate is at least 10 times better.

The sideral SMF rotation period agrees with the results of the spectroscopic measurements of the solar rotation in the interval of latitudes $\theta$ from -40 to 40 degrees. On the contrary at the latitudes between 40 and 55 degrees the SMF rotates faster than other tracers and the photospheric plasma; this results agrees with the solar magnetic field rotating more rigidly at high latitudes. The decrease of the SMF rotation period 

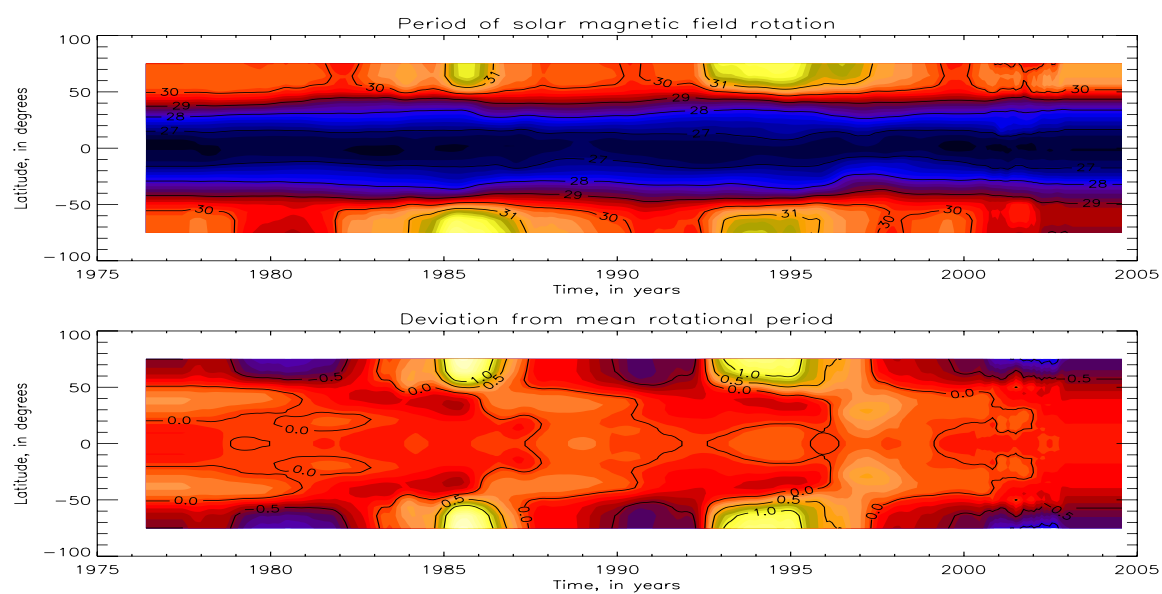

Figure 1. On the upper plot the sideral period of the magnetic field differential rotation is shown as a function of time and latitude. Blue and yellow (dark and light in white-and-black version) colors correspond to the shorter (longer) periods. Contours correspond to the sideral rotational periods of 27, 28, 29, 30 and 31 days. On the bottom plot the mean North-South deviation of the time dependent rotational period from the differential rotation period averaged over 22 years is plotted as a function of time and latitude. Blue and yellow (dark and light) colors correspond to negative and positive deviations. Contours correspond to the deviations of $0, \pm 0.5, \pm 1.0$ days.

at latitudes above 55-60 degrees (deduced from the full 29 years long data sets) was never found for other tracers or in spectroscopic measurements (see, for example, Beck, 1999 and refeerences there) while during some intervals of time the SMF rotation at high latitudes is rigid. This could be attributed to the presence of slow rotating coronal holes.

The radial and latitudinal dependence of the rotational rate was obtained by helioseismological methods, (see for example, Rhodes et al., 1990). The higher rotation rate at the latitudes above the 55 degrees coincides with the rate of the rotation at a deeper layer. This is an evidence that the solar magnetic field rotation at latitudes above 55 degrees follows the rotation of the deeper layers where it is originated from. This result confirms the increase of the rotational rate during the periods and in the latitudinal zones of weak magnetic field and it agrees with the conclusions of Stenflo, 1990; Makarov et al., 1997 and Obridko and Shelting, 2001 that the pre-equatorial SMF rotation rate depends on the phase of the solar cycle and is higher during minimum of the activity.

Results obtained by both methods entirely agree with these conclusions.

I thank the WSO team for the possibility to use the obsevations of the photospheric magnetic field.

\section{References}

Beck, J.G. (1999), Solar Phys., 191, 47

Gavryuseva, E., and G. Godoli (2006), Physics and Chemistry of the Earth, 31, 68

Howard, R.A., and B.J. LaBonte (1980), with a period of 11 years, Astrophys.J., 239, L33

Makarov, V.I., A.G. Tlatov, and D.K. Callebaut (1997), Solar Phys., 170, 373

Obridko, V.N., and B.D. Shelting (2001), Solar Phys., 201, 1

Rhodes, E.J., Jr., A. Cacciani, S. Korzennik, S. Tomczyk and R. Ulrich (1990), ApJ, 351, 687

Stenflo, J.O. (1990), Astron.Astrophys., 233, 220 